\title{
INVESTIGATION ON METHODS AND CHARACTERISTICS IN MEDICAL DEVICE DEVELOPMENT
}

\author{
J. Kuhl $\bowtie$, O. Sankowski and D. Krause \\ Hamburg University of Technology, Germany \\ $\varangle$ juliane.kuhl@tuhh.de
}

\begin{abstract}
A targeted development of safe medical products can be supported by design methods. This paper analyses which design methods are applied in the development of medical devices and whether they are adapted for considering medical devices' special features (legal, human and technical issues). In particular, variety management, risk assessment and user-centered design for medical devices are examined. Typically, interdisciplinary risk assessment is methodically supported. Additionally, user-centered design methods for requirements assessment, design verification and design validation are applied.
\end{abstract}

Keywords: biomedical design, design methods, risk management, user-centred design, medical device development

\section{Introduction}

The increasing demand for patient safety and security of supply within the medical sector is challenging medical device companies to further optimize and personalize their products. In order to develop high-quality products with low error rates and low risk, different design methods can be applied to support a goal-oriented product development process (Pahl et al., 2007). As safety issues are particularly important for medical devices, the application of design methods within the healthcare sector should be given appropriate importance. However, medical devices have special issues (Lantada and Morgado, 2013; Santos et al., 2013) that may need to be considered when applying design methods in the development process.

The investigation on how these special issues and characteristics of medical devices influence the application of design methods is the subject of this paper. Since the consideration of individual, patient specific requirements within the design of medical devices is increasingly focused (e.g. Maier et al., 2017), special attention is paid to the application of variety management and modularization methods, through which the management of variety-induced complexity can be supported (Simpson et al., 2014; Krause and Gebhardt, 2018).

After the presentation of known special issues of medical devices in section 2, the research method is introduced (section 3). Afterwards, the application of variety management methods for medical devices (section 4.1) as well as the use of general product development methods for medical devices (see section 4.2) is investigated. In Section 4.3 and 4.4, the application of risk assessment as well as user-centered methods for medical devices is emphasised in detail. In doing so, special attention is paid to the extent to which design methods were adapted to make them applicable to medical devices. 
Finally, the results are summarized (section 4.5 and 4.6) and discussed (section 5), before a conclusion is given in section 6 .

\section{Background}

As a background for this paper, the state of the art in the development of medical devices and its special issues and characteristics as well as variety management and user-centered design is presented in the following.

\subsection{Special issues in medical device development}

According to the Regulation (EU) 2017/745 of the European Parliament (2017), the European definition for medical devices is as follows: “'medical device' means any instrument, apparatus, appliance, software, implant, reagent, material or other article intended by the manufacturer to be used, alone or in combination, for human beings for one or more of the following specific medical purposes:

- diagnosis, prevention, monitoring, prediction, prognosis, treatment or alleviation of disease,

- diagnosis, monitoring, treatment, alleviation of, or compensation for, an injury or disability,

- investigation, replacement or modification of the anatomy or of a physiological or pathological process or state,

- providing information by means of in vitro examination of specimens derived from the human body, including organ, blood and tissue donations,

and which does not achieve its principal intended action by pharmacological, immunological or metabolic means, in or on the human body, but which may be assisted in its function by such means." This definition covers a broad range of products, which differ from each other in aspects like risk potential, development time or production volumes (Santos et al., 2013). To classify products in Europe, medical devices are divided into four different risk classes I, IIa, IIb and III (European Parliament, 2017). In a nutshell, the risk class depends on the duration and location of use of the medical device, i.e. whether it is used externally or invasively. The longer and the more contact the product has with tissues in the body, the higher the risk class. Depending on the risk class, medical devices are subject to strict regulations that must be fulfilled in order to be admitted to the market as a medical device. In Europe the conformity of the developed product must be proven by the manufacturer himself. Notified bodies check the declaration of conformity (except for certain products in risk class I) (BVMed, 2016). Both technical as well as clinical factors must be examined within the scope of the conformity assessment. During design verification it is checked, if all technical requirements were considered during the design phase while design validation confirms that the final product fulfils the intended use (Alexander and Clarkson, 2000). If conformity is approved, the medical device can be provided with a CE mark and offered on the market (BVMed, 2016). Taking into account different methodologies for the development of medical devices (e.g. US Food and Drug Administration (FDA), 1997; Santos et al., 2013) and combining it with the general engineering development process of VDI 2221 (Pahl et al., 2007), a medical device development process can be summarized as shown in Figure 1, paying special emphasis to design verification and validation.

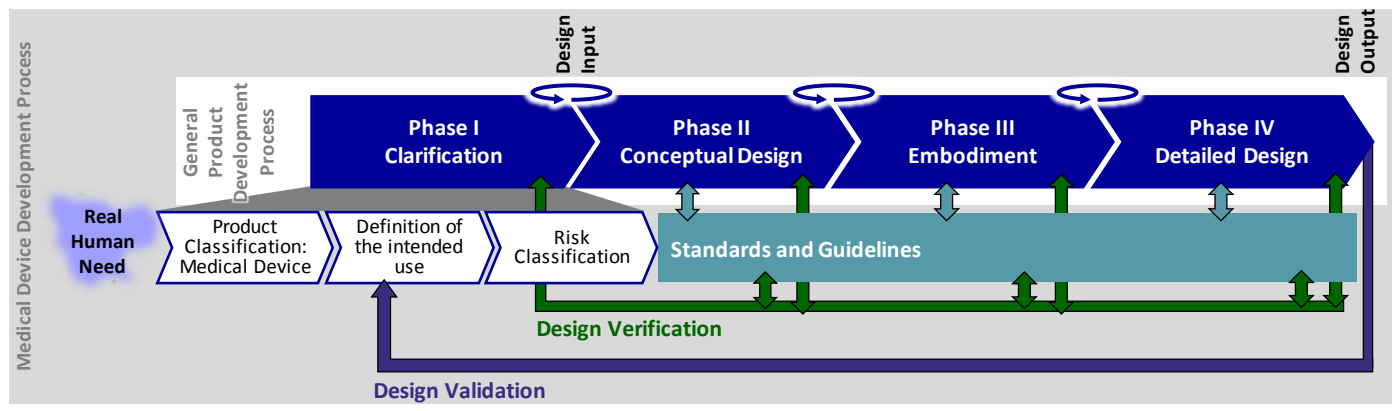

Figure 1. Development process for medical devices 
Legal issues, which include the points just described, also cover ethical issues including beneficence, non-maleficence, justice, and respect for autonomy, which must be taken into account when developing medical devices (Santos et al., 2013). Lantada and Morgado (2013) further add technical and human issues. Technical issues refer to geometric and material details, which have certain characteristics due to the direct contact of the medical device with the human body. These include aspects such as biocompatibility of materials or the cleanable and sterilizable design of the devices. Human issues include the coordination of different experts, who all have an interest in the medical device and can provide different inputs during development (Lantada and Morgado, 2013). The heterogeneity of the stakeholders is also emphasized by Santos et al. (2013). In many cases medical data and databases can be used to specify the use case and clarify the anatomical requirements. On the other side, according to Santos et al. (2013), economic issues are less important. Although, in order to establish itself, a medical device must be competitive when introducing it to the market, it is more important to offer a product with good quality and low risk. Figure 2 summarizes special issues, which have to be considered in medical device development.

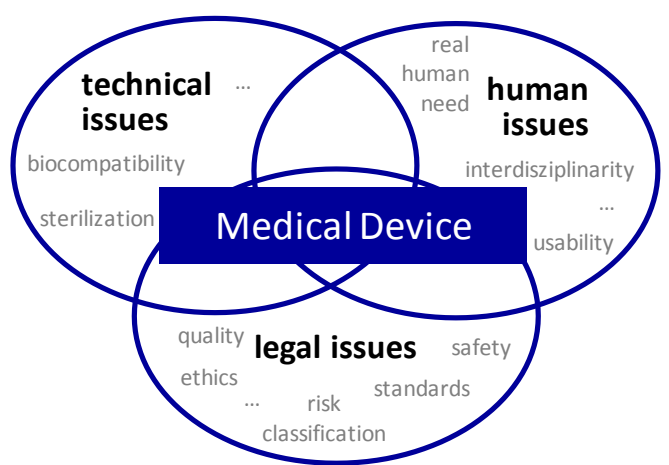

Figure 2. Special issues in medical device development

Despite the many special issues that must be taken into account in the development of a medical device, there is no uniform procedure for the development of medical devices (Azman et al., 2017). Therefore, at this point the question arises why there are no methods and methodologies for the development of medical devices. For answering this question, it is necessary to check whether there are specific methods for the development of medical devices and how they differ.

\subsection{Design methods}

Design methodologies support the development of products and technical systems by proposing a sequence of different design phases through which a solution can be systematically developed (Pahl et al., 2007). One example for a methodology is contained in VDI 2221 (Pahl et al., 2007). Within each phase, different methods can support the systematic solution finding (Pahl et al., 2007). A method is a description of a rulebased and scheduled procedure for obtaining knowledge or results (Krause and Gebhardt, 2018).

\subsubsection{Personalization strategies}

For a better adaption of the medical device to the patient specific anatomies, personalized medical devices become more and more important (Federal Ministry of Education and Research, 2018; Maier et al., 2017). Thus, an optimal functionality of the device for every patient can be reached. Therefore, the consideration of individual, patient specific requirements within the design of medical devices is increasingly focused. However, considering individual requirements within the product development process results in an increase of the variety-induced complexity emerging of the variety of requirements, parts, components, products as well as processes within a company (Simpson et al., 2014). To manage this variety-induced complexity, design for variety methods and modularization approaches can be applied (Simpson et al, 2014; Krause and Gebhardt, 2018). It includes mass customization strategies, which differ in their achievable level of customization (da Silveira et al., 2001). Thereby, personalized modules are combined with standard and variant modules to create 
specific product variants (e.g. Lindemann et al., 2006; Koren et al., 2013). Gräßler (2004) introduces a methodology for the Development of Mass Customized Products.

Beside the product, variety-induced process complexity needs to be managed by adequate methods (Lindemann et al., 2006; Spallek and Krause 2016).

Usually, personalization methods focus on how to manage variety-induced complexity. A systematic analyse on which product properties can be personalized while bringing benefit for the customer and being manageable concerning internal variety at the same time, is rarely supported (Kuhl and Krause, 2018). But especially for personalized medical devices, such a method would be very helpful, to balance risk, benefit and complexity at the same time.

\subsubsection{User-centered design}

For the design of (personalized) medical devices, collecting and analysing requirements of various stakeholders, especially the individual and special requirements of patients, as well their implementation into the design process is of high importance. These goals can be achieved by choosing and applying user-centered approaches, methods or tools. Here, user-centered design (UCD) stands for the whole research field of user-centrism (Sankowski and Krause, 2018). Apart of UCD, further terms exist to describe this research area, e.g. Human-Centered Design, Participatory Design, user research or, as stated above, Design for Usability. Herein a plethora of different methods, approaches and techniques exist to elicit user requirements, e.g. interviews, prototyping, usability tests. UCD methods and tools can be applied in all design phases, generally spoken these are planning and task clarification phase, ideation or concept phase and the testing and evaluation phase. Furthermore, methods and tools can be applied for or with primary users (the target or main users that interact with the product to perform its main function), secondary users (those users that also interact with the product on a regular and intended basis to perform auxiliary or subordinate functions), and tertiary users (those persons that do not directly or unintentionally interact with the product and could be negatively affected by it).

Several researchers proposed categorizations of UCD methods and approaches to enhance goaloriented method selection. Sanders (2006) presents a map of design research, where different methods and approaches are clustered and allocated according to their underlying mindset of user-involvement; depending on whether users are seen as subjects, i.e. reactive informers (expert mindset), such as in Human Factors, Ergonomics or Usability Testing, or as partners in design, i.e. active co-creators (participatory mindset), such as in Co-Creation or Storytelling. A broader overview of UCD methods and tools is described by Sankowski and Krause (2018). Here, every kind of method and tool that supports the elicitation and definition of user and usage requirements is allocated to the UCD research, ranging from standards and guidelines with a weak focus on the user, to virtual simulation techniques and emphatical methods (moderate focus) up to methods and approaches with user involvement, e.g. co-design or usability tests, with a strong focus on individual user needs.

User-centered approaches and especially methods with intensive user participation of different user groups throughout the entire product life-cycle are proven to be helpful in creating innovative, satisfactory, efficient and effective products by involving and addressing diverse users' needs. This is also reported in context of medical devices (e.g. Lettl, 2007; Luna et al., 2016). However, ill-targeted or wrongly applied user centrism may also delay or threaten the outcome of a product development process. It is therefore necessary to understand, which kind of UCD methods, at what point of the process and with participation of which stakeholders are necessary for a targeted product development.

\section{Research method}

In order to support the development of safe and high-quality medical devices, this paper will examine the extent to which design methods exist specifically for medical devices and how these differ from other not specially adapted or created methods. Therefore, a large literature review, using the Scopus database, is conducted which includes the investigation on certain keyword combinations. The search results are limited to English publications within the engineering area. Quotation marks (") around search terms symbolize that the words have to appear together, a star $\left(^{*}\right)$ represents that any characters can be inserted instead, e.g. *medical also includes terms like biomedical. 
First of all, the existing methods in the field of variety management for medical devices are examined (keyword combination: *medical and variety/ complexity/modulari*) and compared with the number of publications in the field of personalization/individualization/ customization (see Figure 3).

The search is then extended to general design methods that are used for medical devices. For this purpose, it was examined which topics are addressed in the product development of medical devices. Topic clusters are created using the VOSviewer software. All keywords, which are named at least three times by the author, are included. All too basic terms, such as design, children or entrepreneurship, are excluded manually. Within the defined clusters, which are shown in Figure 3, publications were examined with regard to the application of design methods.

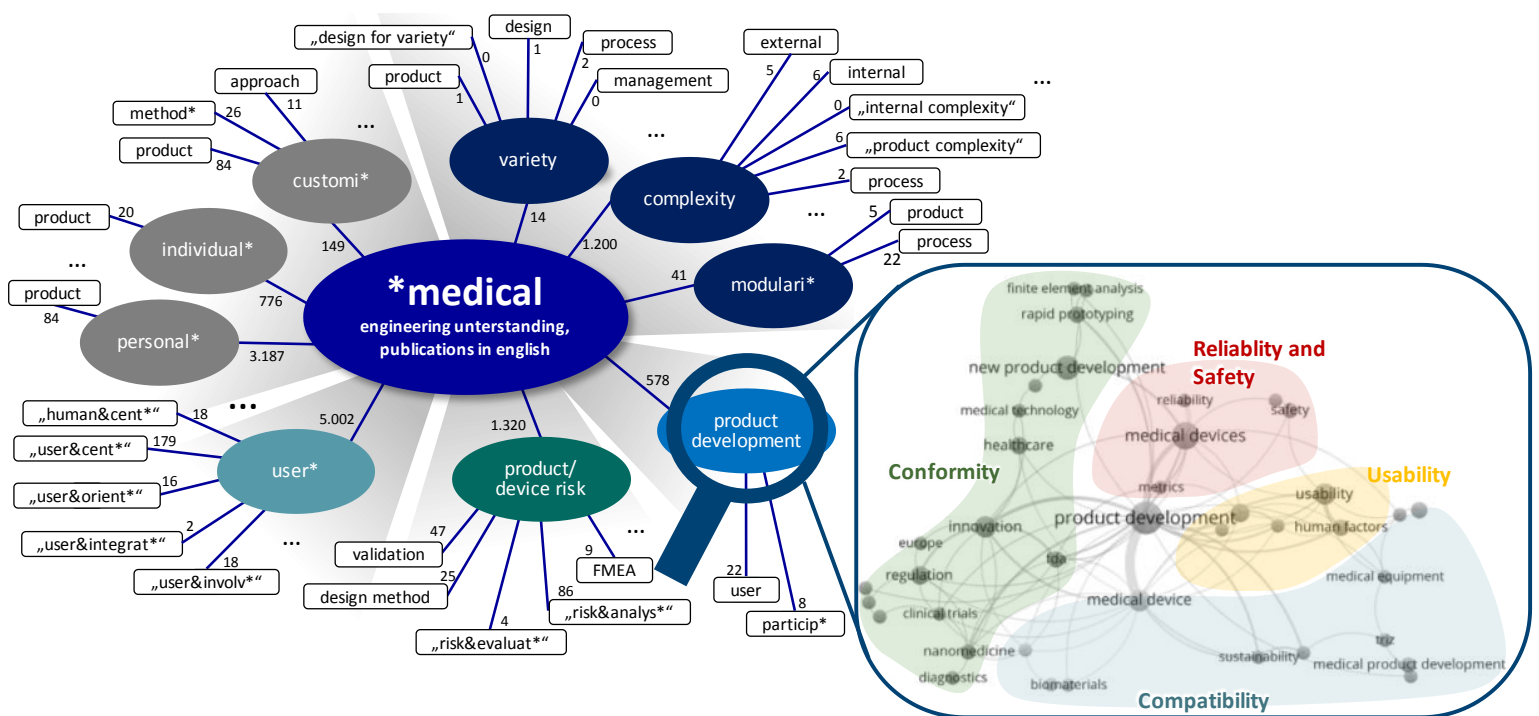

Figure 3. Overview of emphasised keyword combinations and clusters including the number of found publications

The most frequently addressed methods and topics were then analysed (keyword combinations on risk and user*, see Figure 3). Publications that use or present methods to support risk assessment are analysed to identify method adaptations that are associated with the application of methods to medical devices. Since the search for *medical and product risk or device risk resulted in 1.320 hits, the search is narrowed down by adding further keywords including "risk\&evaluat*", "risk\&analys*", validation, design method (see Figure 3, total of 147 hits remain when excluding duplications). In an iterative loop, the keyword FMEA (short form for Failure Mode and Effects Analysis) is added. By first analysing the abstract and then having a closer look on publications, which apply a certain design method, general conclusions can be drawn.

Additionally, publications dealing with any kind of UCD and common synonyms (Human-Centered Design, User Involvement, etc.) are emphasised; without restricting the search to any specific method or approach, such as Design Thinking, Co-Design or User Experience Design. Hits are checked to see if they describe a medical device development case study and whether they are using any type of UCD method or tool. All paper fulfilling both criteria are selected for description and analysation of the state of the art in UCD for medical device development.

Finally, the findings are summarized and their significance for the design of personalized medical devices is addressed. Furthermore, limitations are discussed before the conclusion is reached.

\section{Design of medical devices}

The results of the research procedure described in section 3 are presented below.

\subsection{Design for variety for medical devices}

The large number of publications on keyword combinations dealing with *medical in personalization, customization and individualization prove the close relation of those topics as earlier presented in 
section 1. Although the importance of variety management for an increasing number of variants as in case of personalized product design is known within the engineering design community, the analysis on the keywords shows that the variety management itself is not focused at all in related literature. There is no publication in Scopus database dealing with medical devices and Design for Variety or variety management. Also, the search for keywords closely linked to variety like complexity, modularity or product family, product structure or product architecture show no relevant matches on how to define and manage the number of variants within a medical device product family. Since even the search for variety management named in the title or abstract offers no results, no typical characteristics for medical devices in variety management methods can be summarized.

Therefore, at this point, the question arises why there is no variety management for medical devices. Can standard methods be applied directly for medical devices without changing or adapting them? Or is there an unresolved need for adaptation? In order to answer these questions, it is investigated which methods specified for the use in medical device development exist so far and which adaptations have been made to them.

\subsection{Design methods for medical device development}

The investigation on medical device development topics emphasise, that design validation and verification are of high importance and part of every topic focused in medical device development. This makes clear that one of the main tasks during development is to ensure the safety and usability of the medical device. This can also be proved by having a closer look on methods for medical device development. Different methods can be found that support the design of safe and high-quality products in the medical device sector. Within methods for medical devices, two aspects are nearly always included, both contributing to a high quality and good usability of medical devices:

- risk assessment as part of quality management

- some kind of user involvement method or tool, i.e. UCD approach

Legal and product-related issues of medical devices are implemented in the development process by the methodical evaluation and assessment of risks. For this purpose, different methods and tools are used, which are explained below. Additionally, human issues, that need to be taken into account for medical devices, are also supported by methods and tools. Here, the user-centered design approach is applied, which is also examined in more detail below.

\subsection{Risk assessment for medical devices}

The found publications are further analysed concerning their method application for risk assessment. Special attention is paid to method adaptions and the target of the methods, which are applied for executing the risk assessment within the development of medical devices. It is not possible at this point to name an exact number of publications that use a particular method of risk assessment. Rather, the analysis of the publications reveals generally valid aspects that are sometimes more and sometimes less focused. In many cases the method is hardly emphasized, but rather the application example is focused. Common features that occur particularly frequently are explained below:

Within the found methods, risk management for medical devices is most likely supported by Failure Mode and Effects Analysis (FMEA) (e.g. Liu et al., 2012; Wang et al., 2019). Hereby, possible product defects are tried to be estimated and possible consequences are assessed by the company. Searching for *medical in combination with FMEA, further published and applied methods can be analysed concerning their method adaptions. For medical products, the general approach can be applied as usual, including the definition of occurrence, impact and prevention factors by the engineer. For supporting the assessment of the exact factors, some publications provide different tables and checklists with indicators directly related to the clinical situation (e.g. assessment of urgency or stress) (e.g. Liu et al., 2012; Catelani et al., 2014).

Besides using tables resp. checklists, the rating of the occurrence, impact and prevention factors should not be done only by the company, but by different experts with medical and technical background. Thus, the risk analysis becomes more realistic since expertise of both disciplines is integrated. For expressing the risk factors, it is suggested to not use crisp numbers but linguistic terms, 
like very low, medium or high, to express the failure modes, which help to integrate the mental behaviour of the rating person.

With interdisciplinary FMEA supported by different checklists, the company pays special attention not only to product failures but also to human factors and human failures occurring during the use phase. But not only the use phase should be evaluated in risk management. Possible failures of the whole product life cycle should be included and checked within the risk management to evaluate an overall performance of a product. Within product development, at each milestone, risk management should be integrated and requirements verified repeatedly.

Among other things, material checklists can for example support the detailed design and avoid failures concerning biocompatibility and sustainability. Simulations can help with identifying failure modes for the load and design of the medical device. Nevertheless, design validations should be performed in practical tests which are carried out in an appropriate validation environment with the device user, as errors can be best identified in this way.

To sum up, particular for the risk assessment for medical devices is the high degree of user integration and the intensive assessment of human failures.

\subsection{User-centered design for medical devices}

It is clear from the methods applied in risk assessment that interdisciplinary work is of great importance in the field of medical device development. In particular, the user of the medical device is involved. User-centered design methods can be applied here. Therefore, UCD for medical devices will be analysed in more detail in the following. One of the main points of interest is to what extent which user is involved in which UCD method.

Publications found through the Scopus search are further analysed for the actual state of the art of UCD methods, their point of application and the users involved in medical device development. The results shall help to conclude recommendations for the design of personalized medical devices.

Excluding the duplications from the hit list, the remaining number of relevant papers from the UCD cluster are 142. Herein, in 114 papers a UCD method or tool is applied. In 48 papers a case study of a medical device development is described. Reducing the list of papers to case studies is necessary since only already applied and proven methods shall be included in the analysis. Keeping all publications dealing with UCD method application as well as a case study, 39 publications remain. However, none of these covers actually a personalized medical device. Figure 4 combines both in section 2.2 presented UCD categorisations in one, while collecting and counting all methods, tools and approaches within the remaining 39 papers. For instance, in 8 of the 39 papers an interview technique is applied. It shall be explained here that in most of the case studies more than one method, tool or approach is named; they are counted individually.

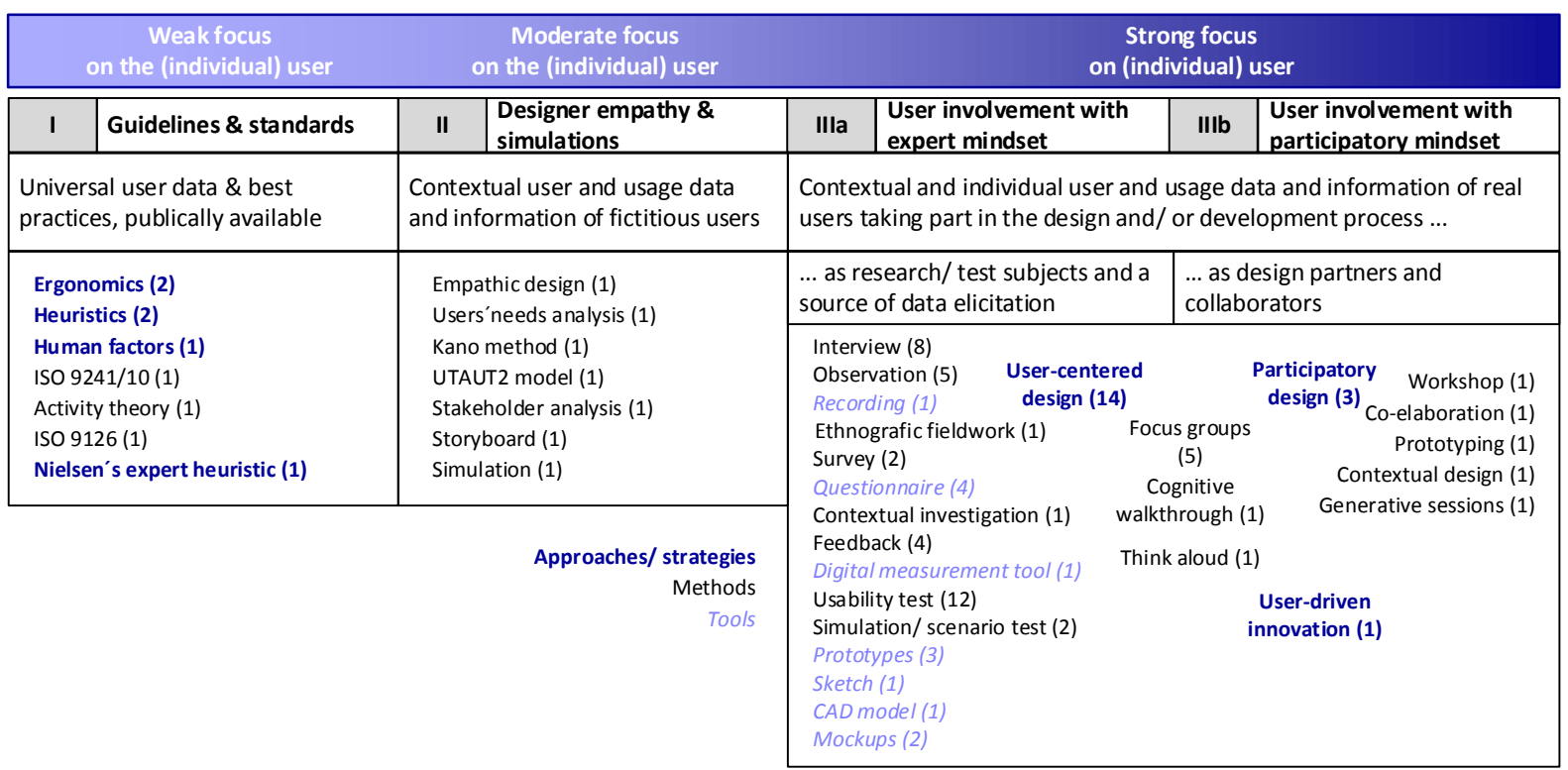

Figure 4. Overview of UCD approaches, methods and tools in medical device development 
Some of the approaches, methods and tools collected cannot be easily allocated into the sub-categories of expert and participatory mindset and are therefore placed in between of both ends. This applies for example for the user-centered design approach, which in theory can be applied with both mindsets, but in most of the papers it is used with an expert mindset.

By collecting, allocating and counting the methods mentioned, a strong focus on the individual user with an expert mindset can be seen in most case studies. Indeed, only very few papers do not mention any kind of user involvement at all, i.e. weak or moderate user focus. In most of the cases, primary users, i.e. medical and clinical staff (15) as well as patients (10), are involved. In a few cases also secondary users, i.e. relatives and informal caregivers (3) are taking part in the design process, but only on behalf of primary users. There is no mention of tertiary users. In almost all of the case studies users are involved for the testing and evaluation of prototypes. This is further confirmed by the number of UCD methods and tools related to testing, such as usability or scenario tests, feedback, survey, questionnaires, measurement tools and all kinds of prototypes. Also, users are often involved in the fuzzy front end of development, i.e. the planning and task clarification phase. Methods and tools related to this phase are: interviews, observations, investigations, fieldwork, thinking aloud and cognitive walkthrough. Only in a few case studies (5) users were involved in the concept phase.

Looking from the literature results, an already strong focus on individual users in design and development of (non-personalized) medical devices can be seen. No especially for the development of medical devices designed or adapted UCD method or tool was found inside of the results. In context of medical devices, where various users, such as patients, relatives, caregivers, prosthetists and physicians, may interact with the device on a regular basis, the involvement of secondary and maybe also tertiary users' needs become increasingly important.

\subsection{Conclusion on method adaptions for medical device development}

Many of the methods applied for medical devices deal with the risk assessment of medical devices. What is repeatedly taken up in the methods is the fact that, in addition to theoretical considerations, practical validation tests must be carried out to detect errors. The same result can be seen in the analysis of UCD methods, where testing and evaluation of prototypes with user involvement is often focused. Interdisciplinary work is of great importance. Otherwise checklists should at least be provided to help an engineer to better understand the clinical routine. In summary, the study has shown that, when applying design methods for medical devices, special issues of a medical device are considered as follows:

- legal and technical issues: continuous assessment of risks including product failures as well as human failures in all development phases, especially by practical tests during design validation (product validation and usability validation) and FMEA with user involvement

- human issues: method application within an interdisciplinary team of engineers and user of the medical device; involvement of the primary user in particular during the early stages of the development process including requirements assessment, but also for verification and especially for design validation; at least assistance (e.g. checklists) for engineers when clinical/ medical situations need to be assessed

\subsection{Application of the results for personalized medical devices}

As section 4.1 has shown, there is no variety management for medical devices or no application of variety management for medical devices that has been found through the structured literature review. A personalized medical device means the development or adaptation of the product specifically for the individual patient, so that each patient receives his own specific product variant. As presented in the introduction, an adequate approach to control variety-induced complexity during the development of personalized products is needed.

The literature review shows, that safety issues are of particular importance. Hence, one of the biggest challenges for variety management for medical devices is to ensure the safety of each patient specific product variant. At the same time, in order to meet individual requirements, the degree and amount of user involvement in the development of personalized medical devices has to be further increased. Practically spoken, this means that the design process has to shift to a holistic user involvement with a 
participatory mindset. This includes that primary users are involved as partners over the whole design process, especially in the concept phase, to discover unmet user needs and further increase usability and user experience. Both, the necessity of risk assessment for each product variant and the involvement of each specific user increases the variety-induced complexity. Therefore, with the help of a new method, the search for a compromise between variety-induced complexity, emerging especially of risk assessment and user involvement, and the benefits of an individual adaptation should be supported.

\section{Discussion and limitations}

Overall, it was very difficult to find methods specifically for the development of medical devices. This could be due to the fact that very different products are grouped under the definition. All medical devices have in common that legal issues must be met and high-quality products with proven conformity and low risk must be developed. The results of the paper underline this once again.

Only publications from the Scopus database were used for the research. Although Scopus contains a very large number of peer-reviewed publications, it is possible that some relevant publications are not included. With the help of other databases, further publications not listed in Scopus can be found and analysed to further strengthen the results of the paper. The same limitation results from the fact that maybe not all relevant combinations of keywords have been used and therefore relevant results remained hidden. Particularly in case of the UCD research field, to which numerous approaches and strategies as well as synonyms can be assigned, the search carried out here may not show the true circumstances, since not all researchers, who use a UCD method or approach may also name it that way. In this case too, the search could be extended so that further relevant publications may be found.

Also, many publications presented the application of a method in the development process of a medical device, but did not detail the method adaptations that resulted. However, it may be that small and unobtrusive method adaptations indeed took place in context of medical product development, but were not reported in that detail. Therefore, in many places it was difficult to assess whether an adaptation was due solely to the fact that it was a medical device. Often the medical device is only a use case for one method, which can also be used for other products. This fact can also be found in the methods specified for the application in design of medical devices. Aspects such as comprehensive risk management or the involvement of the user in the development also apply to non-medical products. However, these are explicitly emphasized as an indispensable necessity for medical devices. This is presumably due to the severity of the effects that a defective medical device that has not been developed with the intended purpose in mind will have. Since medical devices serve nothing less than human health, special attention should be paid during development to intensive risk management and sufficient user involvement. These particularities should already be considered in the methods and methodologies that support the development of medical devices.

\section{Conclusion}

In this paper, methods supporting the development of medical devices are examined as well as the extend, to which legal, human and technical issues are addressed. For this purpose, an extensive literature review was conducted, in which different design methods used in medical device development were examined. Taking into account the limitations of the paper, it was shown that in particular risk management and user involvement are methodically supported. Methods of usercentered design show a strong focus on primary users with involved under an expert mindset. These users are particularly involved in the early development phases as well as in testing and evaluation. Methods of risk management show a strong focus on interdisciplinary and test-based assessment of risk factors, usually by means of FMEA. In this way, both product and human failures can be detected. In summary, it can be seen that safety issues are of paramount importance for medical devices. For this reason, both product and usability risks are examined in the risk assessment with user involvement. For personalizing medical devices, the risk and the real benefit of a personalized device needs to be assessed in addition to the management of variety-induced complexity. 


\section{Acknowledgment}

The authors would like to thank the German Federal Ministry of Education and Research for founding this work within the project BELUCCI (13GW0274D) belonging to the program "Individualisierte Medizintechnik 2".

\section{References}

Alexander, K. and Clarkson, P.J. (2000), "Good design practice for medical devices and equipment, Part I: a review of current literature”, Journal of Medical Engineering \& Technology, Vol. 24 No. 1, pp. 5-13. https://doi.org/10.1080/030919000293987

Azman, N. et al. (2017), "Review on Design for Medical Device", MATEC Web Conference, Vol. 135 No. 5, p. 20. https://doi.org/10.1051/matecconf/201713500020

Bundesverband Medizintechnologie (BVMed) (2016): "Der lange Weg eines Medizinprodukts von der Idee bis zur Anwendung am Patienten", Article, 20.01.2016

Catelani, M., Ciani, L. and Risaliti, C. (2014): Risk assessment in the use of medical devices: A proposal to evaluate the impact of the human factor, 2014 IEEE International Symposium on Medical Measurements and Applications (MeMeA), Lisboa, pp. 1-6. https://doi.org/10.1109/MeMeA.2014.6860088

da Silveira, G.J.C., Borenstein, D. and Fogliatto, F.S. (2001), "Mass customization: Literature review and research directions", Internatioanl Journal of Production Economics, Vol. 72 No. 1, pp. 1-13. https://doi.org/10.1016/S0925-5273(00)00079-7

European Parliament (2017), Regulation (EU) 2017/745, Council of 5 April 2017

Gräßler, I. (2004), Kundenindividuelle Massenproduktion. Entwicklung, Vorbereitung der Herstellung, Veränderungs-management, Springer, Heidelberg. https://doi.org/10.1007/978-3-642-18681-3

Kuhl, J. and Krause, D. (2018), "Strategies for Customer Satisfaction and Customer Requirement Fulfillment within the Trend of Individualization", 29th CIRP Design Conference, 8. - 10. Mai 2019, Procedia CIRP, Póvoa de Varzim, https://doi.org/10.1016/j.procir.2019.04.278

Koren, Y. et al. (2013), “Open-architecture products”, In: CIRP Annals Vol. 62 No. 2, pp. 719-729. https://doi.org/10.1016/j.cirp.2013.06.001

Lantada, A.D. and Morgado, P.L. (2013), General Considerations for the Development of Biomedical Devices, In: Lantada, A.L. (Eds.): Handbook on Advanced Design and Manufacturing Technologies for Biomedical Devices, Springer, Boston, pp. 19-47. https://doi.org/10.1007/978-1-4614-6789-2

Lindemann, U., Reichwald, R. and Zäh, M.F. (2006), Individualisierte Produkte - Komplexität beherrschen in Entwicklung und Produktion, Springer, Berlin. https://doi.org/10.1007/3-540-34274-5

Liu, L. et al. (2012), Use-related risk analysis for medical devices based on improved FMEA, WORK: A Journal of Prevention, Assessment \& Rehabilitation, https://doi.org/10.3233/WOR-2012-0976-5860

Luna, D. et al. (2016), "Impact of participatory design for drug-drug interaction alerts. A comparison study between two interfaces", MIE, pp. 68-72.

Lettl, C. (2007), "User involvement competence for radical innovation", Journal of engineering and technology management, Vol. 24 No. 1-2, pp. 53-75. https://doi.org/10.1016/j.jengtecman.2007.01.004

Maier, A. et al. (2017), "Sensing behaviour in healthcare design", In: Proceedings of the 21st International Conference on Engineering Design (ICED 17), Vancouver, Canada, 21-25.08.2017.

Pahl, G. et al. (Eds.) (2007), Engineering Design. A Systematic Approach, Vol. 3, Springer, Berlin. https://doi.org/10.1007/978-1-84628-319-2

Sanders, E.B.N. (2006), Design research in 2006. Design research quarterly, Vol. 1, No.1, pp.1-8.

Sankowski, O. and Krause, D. (2018), "Using Multi-Channel Human-System Interaction for User-Centered Product Design”. Proceedings of ASME 2018 International Mechanical Engineering Congress and Exposition, November 9-15, Pittsburgh, Pennsylvania, USA. https://doi.org/10.1115/IMECE2018-88091

Santos, I.C.T (2013), Product development methodologies: the case of medical devices, Thesis, Faculdade de Engenharia da Universidade do Porto, Portugal

Simpson, T.W. et al. (2014), "Advances in Product Family and Product Platform Design", Methods \& Applications, Springer, New York. https://doi.org/10.1007/978-1-4614-7937-6

Spallek, J. and Krause, D. (2016), "Process Types of Customisation and Personalisation in Design for Additive Manufacturing Applied to Vascular Models”, Procedia CIRP, Vol. 50, pp. 281-286. https://doi.org/10.1016/ j.procir.2016.05.022

US Food and Drug Administration (FDA) (1997), Design control guidance for medical device manufacturers, Food and Drug Administration, Rockville.

Wang, L. et al. (2019), A linguistic risk prioritization approach for failure mode and effects analysis: A case study of medical product development. Quality and Reliability Engineering International, pp. 1735-1752. https://doi.org/10.1002/qre.2472 